\title{
Fast LIPSS based texturing process of dental implants with complex geometries
}

\author{
Leonardo Orazi $(2)^{a, b, *}$, Riccardo Pelaccia ${ }^{a}$, Oleg Mishchenko ${ }^{c}$, Barbara Reggiani ${ }^{a}$, \\ Maksym Pogorielov ${ }^{\mathrm{c}, \mathrm{d}}$ \\ ${ }^{a}$ Department of Sciences and Methods for Engineering, University of Modena and Reggio Emilia, Italy \\ ${ }^{\mathrm{b}}$ ENETECH, University of Modena and Reggio Emilia, Italy \\ ' Osteoplant Research E' Development, Debica, Poland \\ ${ }^{\mathrm{d}}$ Medical Institute, Sumy State University, Ukraine
}

\section{A R T I C L E I N F O}

\section{Article history:}

Available online 16 May 2020

\section{Keywords:}

Laser micro machining

Surface modification

Texture

\begin{abstract}
A B S T R A C T
In the present work, the generation of Laser Induced Periodic Surface Structures (LIPSS) by using femtosecond laser source is investigated in the surface texturing of Ti grade 5 dental implants. The proposed procedure permits a fast treatment of dental implants characterized by complex shape through the combined and synchronous use of a galvo scanner and of workpiece movements. The obtained surface micro- and nanostructures are characterized from a morphological point of view while in-vitro essays are performed to evaluate cellular proliferation. The results indicate the effectiveness of the LIPSS as method to increase cell viability and the efficiency of the proposed procedure to treat complex geometries as dental implants.
\end{abstract}

(c) 2020 CIRP. Published by Elsevier Ltd. All rights reserved.

\section{Introduction}

The manufacturing process of commercial titanium alloys based dental implant made use of advanced CNC single and multi-spindle turning centers (Swiss lathes). These manufacturing lines guarantee high repeatability, high throughput rate and, at the same time, high flexibility to produce dental implants with complex geometries [1].

The high biocompatibility given by the base material of the implant can be furthermore enhanced by means of a proper modification of surface properties. Indeed, clinical studies indicates that early implant failures, commonly attributed to poor wound healing, inflammation and postoperative infections, are strongly reduced for implants with rough surface implants compared to smooth one [2]. The employed methods operate on surface to modify the chemistry [3], the micro-topography [4] or both [5].

In this context, laser texturing [6] is a well-known and promising method and, in the last years, the use of ultrashort laser sources has offered new possibilities of treatment based on the generation of Laser Induced Periodic Surface Structures (LIPSS). When the laser operates with pulse duration from femtoseconds to few picoseconds and in regime of strong ablation, these structures appear as reliefs and grooves on metals, dielectric as well as on polymers. In addition, they are marked by a periodicity $\Lambda$ well below the dimension of the laser focal spot.

Mechanisms governing the LIPSS periodicity and regularity were not completely explained and understood but, in terms of morphological features, they are classified in two main different types:

\footnotetext{
* Corresponding author.

E-mail address: leonardo.orazi@unimore.it (L. Orazi).
}

1. Low Spatial Frequency LIPSS (LSFL) with a period $\Lambda$ between $\lambda / 2$ and $\lambda$, given $\lambda$ the wavelength of the laser beam. LSFL are perpendicularly oriented with respect to the polarization plane.

2. High Spatial Frequency LIPSS (HSFL) with periodicity below $\lambda / 2$ and oriented along the polarization plane.

This classification, combined with the common wavelengths for industrial ultrashort lasers operating on metals, results in a periodicity in the range of $500 \div 900 \mathrm{~nm}$ for LSFL and $100 \div 200 \mathrm{~nm}$ for HSFL, thus overcoming the limits of the diffraction.

One of the main characteristics of the process is its intrinsic simplicity since vacuum conditions are not requested. It is robust: the non-deterministic and quasi-regular structures self generate in an appropriate range of fluence; moreover, pulse overlapping and multiple passes do not cancel or destroy the structures already generated. Given these peculiar characteristics, LIPSS based texturing are currently investigated for tribology applications $[7,8]$, for the improvement of adhesion strength [9] and to control the wettability of different materials for which the linear aspect of the structures permits to tune the properties with respect to the LIPSS direction $[10,11]$.

LIPSS have been moreover investigated to decrease adhesive wear in carbide insert for cutting tools [12] and for the reduction of the drag force in polymer injection molding by texturing the molds surface [13].

LIPSS-based topography and microstructural changes can play an important role in case of biological and biomedical applications. LIPSS textured surfaces demonstrated beneficial effects in controlling bacterial proliferation on stainless steel, largely used in medical devices and food production machinery [14]. The hierarchical structures 
self-generating on metals can moreover decrease the retainment of bio-fouling [15].

Different works can be found on commercially pure Titanium or Ti alloys used in biomedical applications. In [16] the different classes of LIPSS morphology generated on the surface of Ti-6Al- $4 \mathrm{~V}$ titanium alloy were correlated to the viability of Human Mesenchymal Stem cells on.

A detailed analysis that involved both in-vitro and in-vivo tests can be found in [17], where authors treated both Ti-6Al-4 V and commercially pure Zirconium showing a significant increase in the formation of connective tissues and cells proliferation due to the presence of LIPPS.

A comparison between surface processes including different laser texturing strategies is proposed in [18] with the aim to evaluate the adherence of Streptococcus mutans in abutment dental implants. Authors verified that, among them, LIPSS treatment was marked by the strongest reduction of bacteria. However, in the work, only the abutment has been analyzed, not considering the difficulties in processing the entire dental implants surface.

In this context, the aim of the work is to present and to validate a fast surface process strategy to generate LIPSS on real dental implants keeping into account the scalability for a future mass production.

\section{Experimental set-up}

\subsection{Samples preparation}

Specimens were dental implants made of pure Ti grade 5 and were obtained from a bar of $10 \mathrm{~mm}$ in diameter machined by a Tornos CNC multi-spindle lathe. Dental implants were picked from the production line and, for this reason, the surface was in the same status of items currently employed in dental implantology.

Dental implants geometry had a not regular cylinder-conical shape with the threaded part $14 \mathrm{~mm}$ in length, the cylindrical part (thread crest) $4 \mathrm{~mm}$ in diameter, the root of only $2.6 \mathrm{~mm}$ and the thread flanks strongly inclined at $85^{\circ}$.

\subsection{Laser treatment}

Samples were divided in four groups and laser treated by using a Coherent Monaco $60 \mathrm{~W}$ femtosecond laser operating at $1035 \mathrm{~nm}$ and equipped with a galvoscanner head Scanlab Intelliscan 14 and an ftheta lens with a focal length of $100 \mathrm{~mm}$. The spot dimension in the focal plane was $\sim 25 \mu \mathrm{m}$, with a Rayleigh length of about $0.38 \mathrm{~mm}$ and a calculated DOF (Depth Of Focus) of $\pm 0.12 \mathrm{~mm}$. Inside the DOF, the variation of fluence or intensity was less than $\pm 10 \%$.

The laser beam was linearly polarized with the polarization plane lying along the rotation axis.

The system was equipped with a 5 -axis workpiece manipulator used for the relative placement of the workpiece with respect to the scanning head and the rotative movements.

The treatment scheme is shown in Fig. 1, with the dental implant clamped on the spindle with the axis parallel to the focal plane and

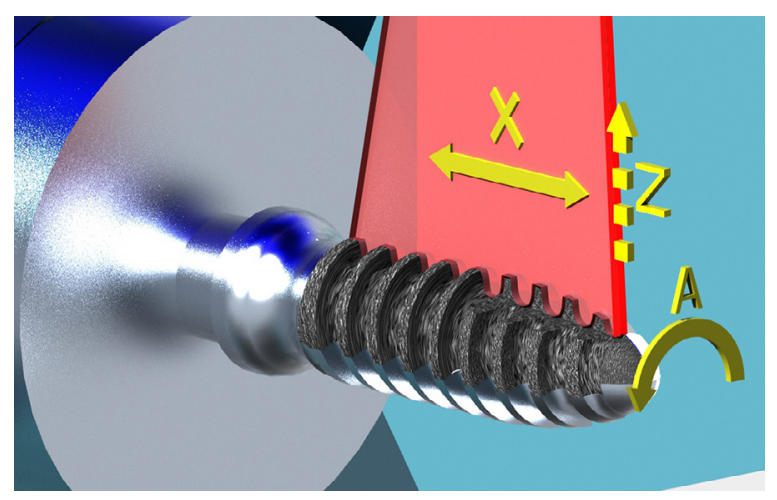

Fig. 1. LIPSS scanning process of the dental implant.
Table 1

Laser processing parameters.

\begin{tabular}{llllll}
\hline Parameter & & & & Value \\
\hline Pulsed duration & $\mathrm{Fs}$ & & & 350 & \\
Pulse frequency & $\mathrm{kHz}$ & & & 4000 & \\
$\begin{array}{l}\text { Scanning Speed } \\
\text { Pulse Energy }\end{array}$ & $\mathrm{mm} / \mathrm{min}$ & & & 2500 & \\
Peak Fluence & $\mu \mathrm{J}$ & & & 1.95 & \\
& $\mathrm{~J} / \mathrm{cm}^{2}$ & & & 0.8 & \\
& & $\mathrm{PA}$ & $\mathrm{PB}$ & $\mathrm{PC}$ & $\mathrm{PD}$ \\
\hline Hatch Space & $\mu \mathrm{m}$ & 10 & 10 & 10 & 5 \\
Focal positions per cycle & & 0 & $0,0.25,-0.25$ & $0,0.25,-0.25$ & $0,0.25,-0.25$ \\
Pass time & $\mathrm{s}$ & 18 & 18 & 18 & 36 \\
$\omega_{r}$ & $\mathrm{rad} / \mathrm{s}$ & 0.35 & 0.35 & 0.35 & .0175 \\
N. Passes & & 1 & 3 & 15 & 3 \\
Total working time & $\mathrm{s}$ & 18 & 54 & 270 & 108 \\
Cumulate dose & $\mathrm{J} / \mathrm{cm}^{2}$ & 79 & 715 & 1192 & 1430 \\
\hline & & & & & \\
\hline
\end{tabular}

the F-theta lens placed at a distance $l_{f}=f+r_{p}$, where $f$ is the focal standoff distance and $r_{p}$ the average implant radius equal to $1.6 \mathrm{~mm}$. The laser beam was repetitively scanned along the rotation axis covering the entire dental implant surface in a single turn without the need to calculate the complicated and time-consuming part program and without the necessity of highly accurate re-positioning procedures.

Due to geometric complexity, only the points lying at radius $r_{p}$ were at the proper focal position. Moreover, the fluence and the specific energy dose requested to obtain LIPSS of good quality are influenced by the relative orientation between the laser beam and the surface normal. Thus, the dental implants were subdivided in four set named PA, PB, PC and PD each of them treated under constant laser parameters by varying the kinematic conditions as described in Table 1.

The hatch conditions were controlled by regulating the rotation speed $\omega_{r}$ with respect to the scanning time for a single vector $\left(\tau_{s}\right)$. At the nominal radius $r_{p}$, the hatch distance was $h_{s}=\omega_{r} \cdot r_{p} \cdot \tau_{s}$.

Single pass was focused at an average radius $r_{p}$ between the outer surfaces on the thread crests and the inner surfaces of the thread roots. In case of 3 passes, the focus was placed at $r_{p}, r_{p}+0.25 \mathrm{~mm}$ and $r_{p}-0.25 \mathrm{~mm}$ for a complete coverage. A test condition with 5 complete cycle repetition ( 3 passes repeated 5 times) was moreover performed to evaluate cumulative effects on LIPSS generation.

The common parameters of Table 1 were determined by preliminary tests on flat surfaces of the same material, centered in the operating window, with the aim to obtain uniform LIPSS.

The same parameters were used to treat disks $10 \mathrm{~mm}$ in diameter for biological assessment.

\subsection{Morphological analysis}

Morphological analyses of the surfaces were conducted on a FEI Nova NANOSEM scanning electron microscope equipped with X-EDS Bruker QUANTAX-200 microprobe. LIPSS periods were investigated through the use of Gwyddion software on calibrated images.

\subsection{Biological assessment}

Cell culture experiments were conducted on disks treated in same regimes of Table 1 (Ti-LIPSS) due to impossibility of cell culture assessment on real shape dental implants. Untreated Ti disks were used as a control (Ti-Untr). All samples were placed in 24-well plate and filled with Dulbecco's Modified Eagle Medium/Nutrient Mixture F-12 (DMEM/F-12) for $24 \mathrm{~h}$. After media removal, $10^{4}$ osteogenic cell line (HOS cells) were seeded in each sample and Alamar blue (Invitrogen) were added in an amount equal to $10 \%$ of the volume to each well. The plates were incubated for $4 \mathrm{~h}$ at $37^{\circ} \mathrm{C}$, in the dark. The absorbance of medium was measured using a Multiskan FC (Thermo Fisher Scientific) plate reader at wavelengths of $570 \mathrm{~nm}$ and $600 \mathrm{~nm}$. The cells were quantified at different time intervals: 1 day, 3 days and 7 days. All experiments were repeated 3 times. The percentage of Alamar blue reduction was calculated according to the manufacturer's protocol. SEM microscopy of samples were made after 7 day of cultivation to obtain information about cell-surface interaction. 


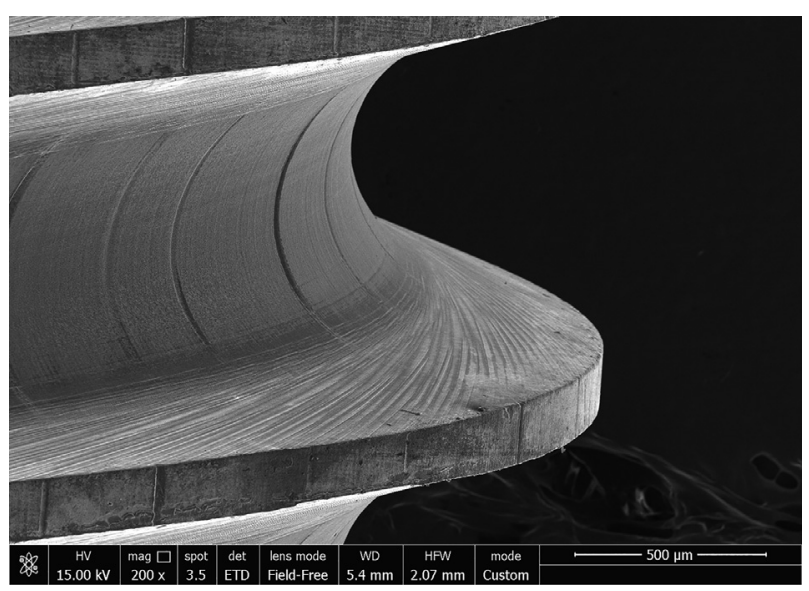

Fig. 2. A screenshot of the threaded part of a dental implant with the marking lines generated by prior machining working phases.

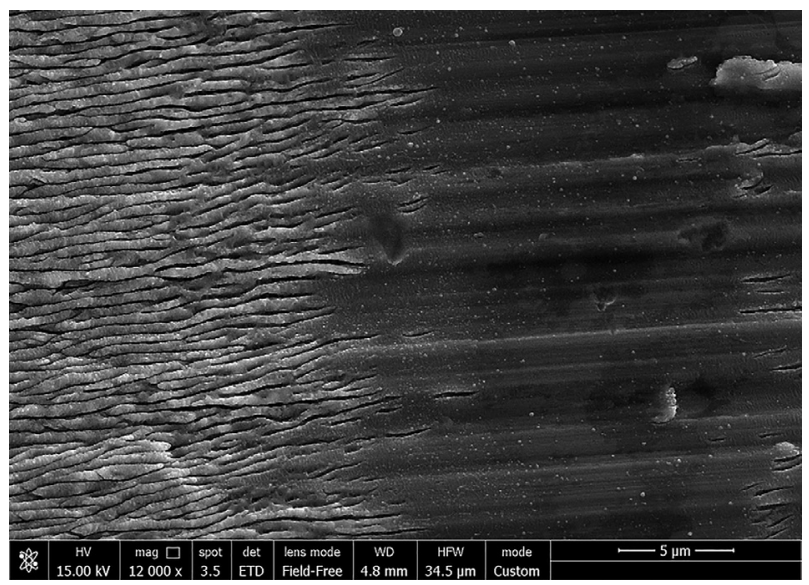

Fig. 3. Partial LIPSS formation on the thread root surface of a PA sample. Only the central part of the gaussian distributed fluence overcome the LIPSS formation threshold.

\section{Results and discussion}

The picture in Fig. 2 shows a low magnified image representing the status of the dental implant surface after the machining working phase. The surface is characterized by well evident machining signs due to the complex cutting toolpaths.

A typical SEM observation of PA samples, treated with a single pass, is shown in Fig. 3.

The image, focused on the thread root, evidences that LIPSS do not completely cover the surface. Indeed, the laser intensity distribution is almost Gaussian and the fluence overcomes the threshold only in the central part of the tracks.

The multi-passes treatment of PB samples is shown in Fig. 4. The increasing of the overall dose and the variation of the focal position during the three steps permits a complete treatment of both thread roots and crests in a single placement.

The nominal dose changes of almost an order of magnitude from the PA treatment $\left(79 \mathrm{~J} / \mathrm{cm}^{2}\right)$ to the PB one $\left(715 \mathrm{~J} / \mathrm{cm}^{2}\right)$. It must be outlined that this calculation does not keep into account local surface orientation and the small defocus due to variation in Z. For this reason, no significant changes in the LIPSS morphology appeared, as

Table 2

Periodicity analysis.

\begin{tabular}{llllll}
\hline & & PA & PB & PC & PD \\
\hline Average $\Lambda$ & {$[\mathrm{nm}]$} & 508 & 459 & 403 & 481 \\
$\Lambda$ Standard Deviation & {$[\mathrm{nm}]$} & 34 & 29 & 19 & 29 \\
\hline
\end{tabular}

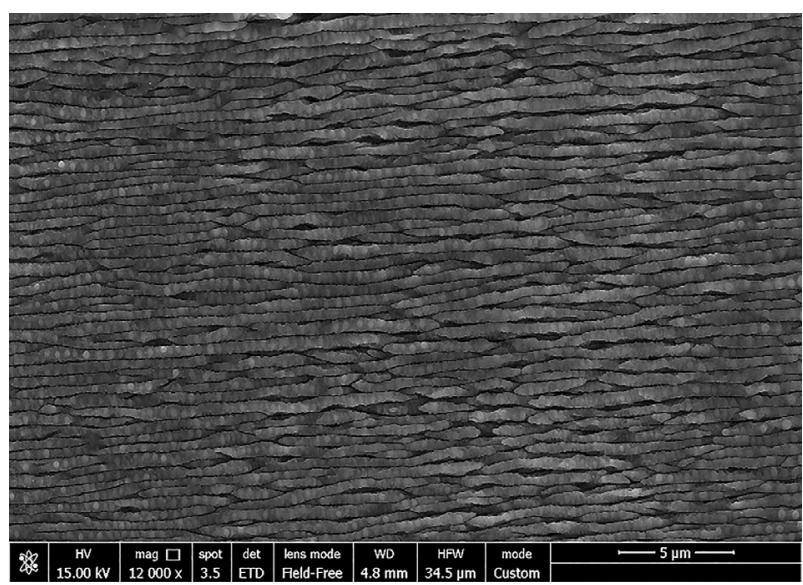

Fig. 4. Uniform and regular LSFL distribution on a PB sample thread root. The same morphology appears on implant thread crests.

reported in other works [14]. LIPSS periodicity $\Lambda$ was investigated by averaging the distance of 10 LIPSS reliefs and repeating the measure 8 times for each treatment. Results are shown in Table 2, highlighting an average LIPPS period of around half a micron and negligible differences between values (accounting for the standard deviation).

The texturing of the thread flank is characterized by a high incidence angle with respect to the laser beam with a decreasing of the real fluence. Fig. 5 shows a detail of what happen on the thread edge where surface orientation abruptly changes respect to the laser beam direction. Image clearly shows that the operating parameters generate uniform LSFL perpendicularly oriented with respect to the polarization plane on the crest. On the highly inclined flank surface, the self-structuring process turns to HSFL oriented along the polarization plane with lower periodicity and height.

Cell culture experiment showed significant better cell adhesion in $24 \mathrm{~h}$ after HOS cultivation (Fig. 6). It is well known that Ti is biocompatible and supports cell proliferation, but untreated surface showed only $62.7 \pm 6.3 \%$ and $82.1 \pm 7.4 \%$ of resazurin reduction compared to $79.4 \pm 3.8 \%$ and $98.3 \pm 1.4 \%$ of LIPSS treated on day 3 and day 7 after cell seeding. The aspects underlying these results were not completely understood from a biological point of view since LIPSS periods are much smaller than average cells dimension. However, the nanopatterns generated on titanium surface could provide additional sites for protein adsorption compared to the untreated ones [17]. These precursors act as sites for the cell processes attachment and many connection points increase the probability of cell growth and proliferation.

SEM images demonstrate that cells on untreated surface have multiply short and long processes widely attached to Ti substrate (Fig. 7a). HOS cells on LIPSS treated surface has only short processes

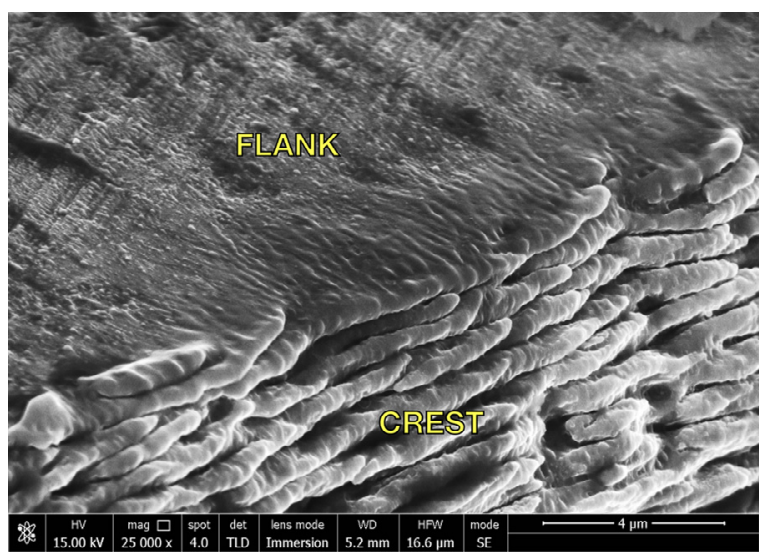

Fig. 5. The external surface of the dental implant thread crest with uniform LSFL. To outline the presence of HSFL on the inclined thread flank (Sample PC). 


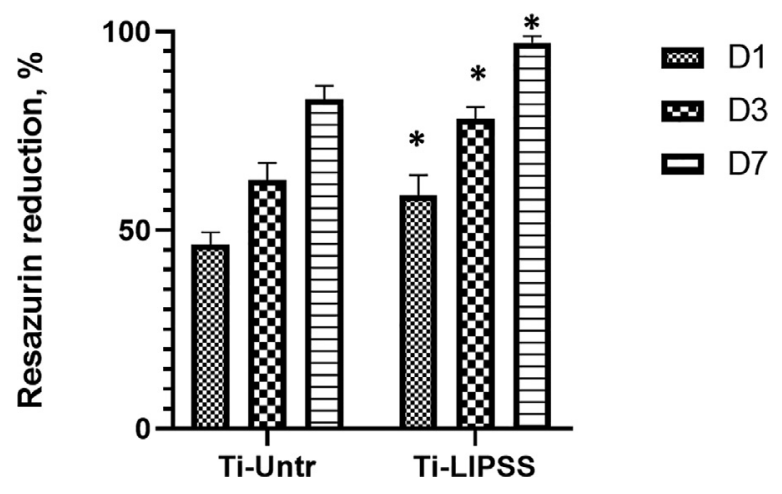

Fig. 6. Resazurin reduction assay during 7 days of HOS cells cultivation on untreated and LIPSS treated surfaces. ${ }^{*}$ - significance between polished and LIPSS surfaces $(p \leq 0.05)$.

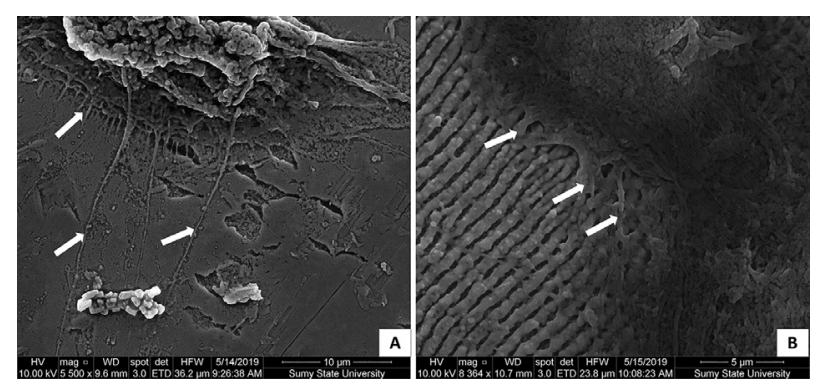

Fig. 7. SEM of cell attachment to polished (a) and LIPSS (b) surfaces. Arrow demonstrate cell process attached to the surface.

attached to nearest nanometric reliefs suggesting less metabolic energy consumption by cell. A favorable cell attachment causes better proliferation rate, especially in the early stage, and could provide successfully osteointegration.

\section{Conclusions}

In the present work, dental implants with complex geometry were treated inducing LIPSS on the surfaces. The micro- and nanopatterns were generated by combining the optical scanning with the continuous rotational movement and with the radial displacement to progressively maintain the entire surface inside the depth of focus. By using this strategy LIPSS based texturing process can be included in the manufacturing chain without the need of specific part programs and the use of expensive 5-axis, high precision manipulator systems. It moreover simplifies parts fixture and alignment. In addition, the low working time (between 18 and 270 s) fulfills the high productivity requirements for mass production. The proposed procedure permits to generate LSFL on all the implant except on the highly inclined thread flanks near the thread crests where HSFL appear. Invitro tests indicate that LIPSS nanopatterned titanium surfaces provide better environment for cell attachment and proliferation compared to untreated ones.

The combination of these results shows that the proposed method can be scaled-up for the mass production of dental implants with advanced osteointegrative properties reducing the probability of implants failure.

\section{Acknowledgements}

Biological investigations provided with support of H2020 MSCARISE NanoSurf 777926 project.

\section{References}

[1] Axinte D, et al. (2019) Machining of biocompatible materials - Recent advances. CIRP Annals 68(2):629-652.

[2] Oh S-L, Shiau HJ, Reynolds MA (2020) Survival of Dental Implants at Sites After Implant Failure: A Systematic Review. The Journal of Prosthetic Dentistry 123 (1):54-60.

[3] Neto CLBG, da Silva MAM, Alves C (2009) Osseointegration Evaluation of Plasma Nitrided Titanium Implants. Surface Engineering 25(6):434-439.

[4] Elias CN, et al. (2002) Titanium Dental Implants with Different Morphologies. Surface Engineering 18(1):46-49.

[5] Katahira K, et al. (2016) Generation of Bio-Compatible Titanium Alloy Surfaces by Laser-Induced Wet Treatment. CIRP Annals 65(1):237-240.

[6] Sadeghi M, et al. (2019) Role of Micro-Dimple Array Geometry on the Biological And Tribological Performance of Ti6Al4V for Biomedical Applications. Surface and Coatings Technology 362:282-292.

[7] Gnilitskyi I, et al. (2016) Nano Patterning of AISI 316 L Stainless Steel with Nonlinear Laser Lithography: Sliding Under Dry and Oil-Lubricated Conditions. Tribology International 99:67-76.

[8] Kunz C, et al. (2020) Tribological Performance of Metal-Reinforced Ceramic Composites Selectively Structured with Femtosecond Laser-Induced Periodic Surface Structures. Applied Surface Science 499:143917.

[9] Rotella G, et al. (2017) Innovative High-Speed Femtosecond Laser Nano-Patterning for Improved Adhesive Bonding of Ti6Al4V Titanium Alloy. CIRP Journal of Manufacturing Science and Technology 18:101-106.

[10] Florian C, et al. (2018) Controlling the Wettability of Steel Surfaces Processed with Femtosecond Laser Pulses. ACS Applied Materials E Interfaces 10(42):3656436571

[11] Orazi L, et al. (2015) Nonlinear Laser Lithography to Control Surface Properties of Stainless Steel. CIRP Annals - Manufacturing Technology 64(1):193-196.

[12] Zhang K, et al. (2015) Effect of Nano-Scale Textures on Cutting Performance of WC/Co-based Ti55Al45N Coated Tools in Dry Cutting. International Journal of Refractory Metals and Hard Materials 51:35-49.

[13] Sorgato M, et al. (2018) Effect of Different Laser-Induced Periodic Surface Structures on Polymer Slip in PET Injection Moulding. CIRP Annals 67(1):575-578.

[14] Lutey AHA, et al. (2018) Towards Laser-Textured Antibacterial Surfaces. Scientific Reports 8(1):10112.

[15] Rajab FH, et al. (2018) Picosecond laser treatment production of hierarchical structured stainless steel to reduce bacterial fouling. Food and Bioproducts Processing 109:29-40.

[16] Cunha A, et al. (2015) Human Mesenchymal Stem Cell Behavior on Femtosecond Laser-Textured Ti-6Al-4V Surfaces. Nanomedicine 10(5):725-739.

[17] Gnilitskyi I, et al. (2019) Cell and Tissue Response to Nanotextured Ti6Al4V and Z Implants Using High-Speed Femtosecond Laser-Induced Periodic Surface Structures. Nanomedicine: Nanotechnology, Biology and Medicine 21:102036.

[18] Uhlmann E, et al. (2018) The Effects of Laser Microtexturing of Biomedical Grade 5 Ti-6Al-4V Dental Implants (Abutment) on Biofilm Formation. Procedia CIRP 68:184-189. 